\title{
A Simple Construction of Initial Data for Multiple Black Holes
}

\author{
Steven Brandt* and Bernd Brügmann ${ }^{\dagger}$ \\ Max-Planck-Institut für Gravitationsphysik, Schlaatzweg 1, 14473 Potsdam, Germany
}

(Received 11 February 1997)

\begin{abstract}
We consider the initial data problem for several black holes in vacuum with arbitrary momenta and spins on a three space with punctures. We compactify the internal asymptotically flat regions to obtain a computational domain without inner boundaries. When treated numerically, this leads to a significant simplification over the conventional approach which is based on throats and isometry conditions. In this new setting it is possible to obtain existence and uniqueness of solutions to the Hamiltonian constraint. [S0031-9007(97)03144-X]
\end{abstract}

PACS numbers: 04.20.Ex, 04.25.Dm, 04.70.Bw, 95.30.Sf

Binary black hole spacetimes are one of the great challenges for numerical general relativity, even if no matter sources are present. Here we consider the problem of finding initial data for several black holes in vacuum with arbitrary momenta and spins. In general relativity, initial data on a hypersurface cannot be specified freely, because the Einstein equations give rise to four equations, three momentum constraints, and the Hamiltonian constraint that the initial data has to satisfy. The purpose of this Letter is to introduce a novel approach which is significantly simpler than the conventional method based on throats and conformal imaging.

In all that follows we will assume vacuum, that the metric is conformally flat, and that the extrinsic curvature is tracefree. A convenient form of the constraints of general relativity can be obtained by rescaling the physical three-metric $g_{a b}^{p h}$ and its extrinsic curvature $K_{a b}^{p h}$ by a conformal factor $\psi$,

$$
g_{a b}^{p h}=\psi^{4} g_{a b}, \quad K_{a b}^{p h}=\psi^{-2} K_{a b} .
$$

The momentum constraint becomes

$$
\nabla_{a} K^{a b}=0,
$$

and the Hamiltonian constraint becomes an elliptic equation for the scalar field $\psi$,

$$
\Delta \psi+\frac{1}{8} K^{a b} K_{a b} \psi^{-7}=0,
$$

where the covariant derivatives are defined by the flat metric $g_{a b}$, which is also used to raise and lower indices (see $[1,2]$ ).

In order to obtain black hole vacuum data, one has to introduce a nontrivial topology. The first calculations were performed by Einstein and Rosen [3] in their work on point particles in general relativity. Various constructions for black holes based on Einstein-Rosen bridges and "wormholes" were given in, e.g., [4-7]. The spatial slice typically consists of two or more copies of $R^{3}$ with several spheres removed and identifications of the various spherical inner boundaries. In this way several asymptotically flat regions are obtained that are connected by bridges or "throats."
The simplest example derives from the Schwarzschild spacetime in quasi-isotropic coordinates. Considered as a problem on $R^{3}$ minus the point $r=0$, the constraint equations (2) and (3) are solved by

$$
\psi=1+\frac{m}{2 r}, \quad K_{a b}=0,
$$

where $m$ is the mass and $r$ the isotropic radius. To make contact with the throat picture, recall that there exists an isometry given by $r \rightarrow m^{2} / 4 r$ which leaves the coordinate sphere $r=m / 2$ invariant and which maps the entire exterior asymptotically flat space into that sphere. Consequently, there exists a second asymptotically flat region near $r=0$. Equivalently, one can represent this solution to the constraints on a space consisting of two copies of $R^{3}$ with a sphere excised and appropriate identification at the spheres.

For $N$ black holes and nonvanishing extrinsic curvature, York and others [8] have developed a sophisticated method to solve the constraints for two asymptotically flat spaces that are connected by as many throats (i.e., excised spheres) as there are black holes, and that are isometric copies of each other. Note that there are explicit solutions to the momentum constraint (2) that characterize a single black hole with given momentum $P^{a}$, and spin $S^{a}$. For example,

$$
\begin{aligned}
K_{P S}^{a b}= & \frac{3}{2 r^{2}}\left[P^{a} n^{b}+P^{b} n^{a}-\left(g^{a b}-n^{a} n^{b}\right) P^{c} n_{c}\right] \\
& +\frac{3}{r^{3}}\left(\epsilon^{a c d} S_{c} n_{d} n^{b}+\epsilon^{b c d} S_{c} n_{d} n^{a}\right)
\end{aligned}
$$

where $n^{a}$ is the radial normal vector. Since the conformal metric is flat, we will use in what follows either standard spherical or Cartesian coordinates, i.e., $r=\left(x^{2}+y^{2}+\right.$ $\left.z^{2}\right)^{1 / 2}$ and $n^{a}=x^{a} / r$.

By the method of images it is possible to obtain an infinite series based on (5) for $K^{a b}$ which solves the momentum constraint and satisfies an isometry condition at any number of spheres $[6,8]$. Given such a solution, what remains to be done is to solve the Hamiltonian constraint (3), which is an elliptic equation on $R^{3}$ minus several 
spheres, with the inner boundary given by the isometry and the outer boundary determined by asymptotic flatness.

Three independent numerical implementations of the above scheme have been given and compared in the definitive paper on three-dimensional black hole initial data by Cook, Choptuik, Dubal, Klasky, Matzner, and Oliveira [9]. For the Hamiltonian constraint, they consider a nonlinear block full approximation storage multigrid scheme for Cadez coordinates, a successive over-relaxation scheme in Cartesian coordinates, and a multiquadratics approach. All these approaches are greatly complicated by the presence of the inner spherical boundaries and the isometry condition.

Let us return to the Schwarzschild solution of the constraints on a "punctured" $R^{3}$. As noted by Misner and Wheeler [5] and studied in detail by Brill and Lindquist [7] (MWBL), the Schwarzschild solution to the constraints generalizes trivially to $N$ black holes for time symmetry,

$$
\psi=1+\sum_{i=1}^{N} \frac{m_{(i)}}{2\left|\vec{r}-\vec{r}_{(i)}\right|}, \quad K_{a b}=0,
$$

where $m_{(i)}$ characterizes the mass of the $i$ th black hole (i.e., the ADM mass is $\left.\sum m_{(i)}\right)$ and $\vec{r}_{(i)}$ is the location of the $i$ th black hole. For regularity of the conformal factor, the MWBL solution is considered on a single $R^{3}$ with the points $\vec{r}=\vec{r}_{(i)}$ removed. We refer to the $\vec{r}_{(i)}$ as punctures. The isometry present in the Schwarzschild solution is lost, although there still exist minimal surfaces characterizing the throats [7].

Let us now discuss the method that we propose to find data for multiple black holes with arbitrary boosts and spins. The idea is to compactify the internal asymptotically flat regions in order to obtain a simple domain of integration. Such compactification brings up issues of regularity of the fields (e.g., [10,11]), which we address below.

As before, we consider vacuum spacetimes, the metric is conformally flat, and the extrinsic curvature is tracefree. As the spatial slice we choose a single $R^{3}$ with $N$ punctures as in the MWBL data. First, we solve the momentum constraint (2) by setting

$$
K^{a b}=\sum_{i=1}^{N} K_{P S(i)}^{a b},
$$

where each term is defined by (5) with its own origin $\vec{r}_{(i)}$, momentum $\vec{P}_{(i)}$, and spin $\vec{S}_{(i)}$. These parameters correspond to the ADM quantities in the limit that the separation of the holes is very large. Equation (7) defines the solution to the momentum constraint that we actually use, and is not just the starting point for the method of images that is usually invoked to distort $K_{a b}$ to obtain an isometric solution (cf. [12], where the same simplification arises for a trapped surface boundary condition at the inner boundary).
Given $K_{a b}$ as defined in (7), we proceed to solve the Hamiltonian constraint, (3). We rewrite the conformal factor in terms of functions $\alpha$ and $u$ given by

$$
\psi=\frac{1}{\alpha}+u, \quad \frac{1}{\alpha}=\sum_{i=1}^{N} \frac{m_{(i)}}{2\left|\vec{r}-\vec{r}_{(i)}\right|} .
$$

On the punctured $R^{3}$, the Laplacian of $1 / \alpha$ is zero, so that the Hamiltonian constraint equation becomes (cf. [11] for a single asymptotic region and vanishing linear momentum)

$$
\begin{gathered}
\Delta u+\beta(1+\alpha u)^{-7}=0, \\
\beta=\frac{1}{8} \alpha^{7} K^{a b} K_{a b} .
\end{gathered}
$$

To complete the definition of the problem, we have to specify boundary conditions for $u$. For asymptotic flatness at infinity we require $u-1=O\left(r^{-1}\right)$ for large distances to the punctures.

The key question that remains is what condition we want to impose on $u$ close to the punctures. As it turns out, to build in asymptotically flat regions as are present in the MWBL data near the punctures, it suffices to solve (9) everywhere on $R^{3}$ without any points excised. This completes the statement of our proposal.

Let us discuss existence and uniqueness of solutions to the modified Hamiltonian constraint equation (9) on $R^{3}$. Since in this case the topology is trivial, we can show existence and uniqueness of a $C^{2}$ solution by repeating the proof given in [13] for the conventional Hamiltonian constraint equation on $R^{3}$. By definition, both $\alpha$ and $\beta$ are proportional to $\left|\vec{r}-\vec{r}_{(i)}\right|$ near the former punctures and are therefore $C^{0}$, despite the fact that $K_{a b} K^{a b}$ goes as $\left|\vec{r}-\vec{r}_{(i)}\right|^{-6}$ at these points. From the maximum principle and the outer boundary condition we obtain that there exists at most one $C^{2}$ solution, and, in particular, that $u \geq 1$ and $1+\alpha u \geq 1$. Referring to [13] for the definition of weighted Sobolev spaces, for the existence part of the proof we require that $u \in M_{s, \delta}^{p}$ and $\beta(1+\alpha u)^{-7} \in M_{s-2, \delta+2}^{p}$ with $p>3$ for the norm and $s \geq 3$ characterizing differentiability. The falloff of (9) is the same as in the standard case. Interior points like the $\vec{r}_{(i)}$ do not affect the weight $\delta$. Because of the loss of differentiability at the punctures in $\beta$, we find that in our case $p>3$ and $s=3$. (In comparison, [14] uses $p=2$ but requires $s \geq 4$.) The proof proceeds as in [13] with minor changes in the algebra and due care whenever it matters that $\alpha$ and $\beta$ may vanish. So, although $u$ is only $C^{2}$ at the $\vec{r}_{(i)}$, there exists a unique solution for the conformal factor $\psi=u+1 / \alpha$ on the punctured $R^{3}$ determined by our proposal for $u$ on the unpunctured $R^{3}$.

Given a solution $u$, we can demonstrate that each puncture represents the "point at infinity" for another asymptotically flat spacetime (cf. [7]). Hence, solving 
(9) on $R^{3}$ involves a particular compactification of $N$ out of $N+1$ asymptotically flat regions, one of which is distinguished by our choice of $K_{a b}$; see below. We perform a coordinate inversion through a sphere near the $i$ th puncture, $\bar{r}=a^{2} / r$, under which the metric transforms as

$$
d s^{2}=\psi^{4}\left(d r^{2}+r^{2} d \Omega^{2}\right)=\bar{\psi}^{4}\left(d \bar{r}^{2}+\bar{r}^{2} d \Omega^{2}\right),
$$

with $\bar{\psi}=\psi r / a$ (which is not an isometry). Setting $a=m_{(i)} / 2$ where $m_{(i)}$ is the bare mass of the puncture we are considering, we obtain for our choice of $\psi,(8)$, that

$$
\begin{aligned}
\bar{\psi} & =1+\frac{\bar{m}_{(i)}}{2 \bar{r}}+O\left(\frac{1}{\bar{r}^{2}}\right), \\
\bar{m}_{(i)} & =m_{(i)}\left(u\left(\vec{r}_{(i)}\right)+\sum_{j \neq i} \frac{m_{(j)}}{2\left|\vec{r}_{(i)}-\vec{r}_{(j)}\right|}\right) .
\end{aligned}
$$

Therefore, the metric becomes flat as we approach the punctures.

To show asymptotic flatness at the punctures, it remains to be shown that $\bar{K}_{a b}=O\left(\bar{r}^{-2}\right)$. In the physical variables, we just have the coordinate transformation $\bar{r}=$ $a^{2} / r, \quad \Lambda_{c}^{b}=\partial x^{b} / \partial \bar{x}^{c}=\left(a^{2} / \bar{r}^{2}\right) L_{c}^{b}$ where $L_{c}^{b}=\delta_{c}^{b}-$ $2 n^{b} n_{c}$. In the unphysical variables, we also have to take into account the transformation of $\psi$. With $K_{a b}=\bar{\psi}^{2} \bar{K}_{a b}^{p h}$ and $\bar{K}_{a b}^{p h}=\left(\Lambda K^{p h}\right)_{a b}$, we obtain

$$
\bar{K}_{a b}=\left(\frac{a}{\bar{r}}\right)^{6}(L K)_{a b} .
$$

Therefore, for our choice of $K_{a b}$, (5), the momentum term of order $r^{-2}$ is mapped to order $\bar{r}^{-4}$ while the spin term of order $r^{-3}$ is mapped to order $\bar{r}^{-3}$. This observation extends to multiple hole data with $K_{a b}$ defined in (7). Hence the black hole puncture data are asymptotically flat at the punctures.

In fact, we also learn that the black hole seen from the region near the puncture appears to be unboosted, because the boost term is obtained from the $r^{-4}$ term in the untransformed space. This term is always zero in our space by construction. In the conventional method, imposing the isometry leads to terms which go as $r^{-4}$ in $K_{a b}$. These terms may not be included in our method (without modification), because $\beta$ would not be regular at the punctures if they were.

Since the initial data are asymptotic to Schwarzschild data near the punctures, and since small spherical surfaces centered at the puncture of Schwarzschild data are outertrapped surfaces (with outside referring to the asymptotic region away from the punctures), this indicates that the region near the punctures is inside black holes. As in [7], several punctures may be hidden behind a common horizon, which we confirmed numerically but will not discuss here.

We now come to the numerical implementation of our proposal. We found that a full approximation storage multigrid method built around a nonlinear Gauss-Seidel relaxation scheme [15] performs very well for (9) on a finite Cartesian grid with a Robin boundary condition. Note that the standard representation of $\Delta u$ by centered finite differences is only first order at the punctures. One could use a different prescription at the puncture points, but in numerical tests we found that the correspondingly lower rate of convergence is contained in a surprisingly small neighborhood of the puncture points, whether those are part of the grid or not.

The multigrid program is part of BAM, a bifunctional adaptive mesh package for elliptic and hyperbolic problems in three-dimensional numerical relativity (see [16] for the hyperbolic part; we do not use adaptivity here). Since in general the Hamiltonian constraint has to be solved numerically, it is important to provide at least one efficient numerical implementation. There is no reason to believe that the multigrid method is the only good method. However, when available, multigrid methods have proven to be among the best performers. We consider the absence of irregular boundaries in our method to be a valuable feature since it makes a straightforward multigrid implementation possible.

As a test, let us compute the correction to the conformal factor for two equal mass black holes boosted toward one another when we keep only terms of small $P$ (the momentum of each hole) and $L$ (the coordinate positions of the holes along the $z$ axis being $\pm L$ ). Consider $\beta$ to be of order $\epsilon$, and consider the MWBL solution to be the zeroth order solution to the Hamiltonian constraint, $u=u_{(0)}+\epsilon u_{(1)}=1+\epsilon u_{(1)}$. We now have

$$
\epsilon \Delta u_{(1)}=-\beta(1+\alpha)^{-7} \text {. }
$$

The ADM mass to first order in $\epsilon$ is

$$
\begin{aligned}
M_{\mathrm{ADM}}= & -\frac{1}{2 \pi} \oint_{r=\infty} \nabla_{a}\left(\frac{1}{\alpha}\right) d S^{a} \\
& +\frac{1}{2 \pi} \int \beta(1+\alpha)^{-7} d V \\
= & 2 m+m\left(\frac{P}{m}\right)^{2}\left[\frac{11}{50}\left(\frac{L}{m}\right)^{2}-\frac{24}{35}\left(\frac{L}{m}\right)^{4}\right] .
\end{aligned}
$$

This equation predicts the correction to the ADM mass resulting from the full nonlinear solve to within about $5 \%$ when $P / m \leq 1.0$ and $L / m \leq 0.2$ and the grid is a cube whose sides have a length of about $16 \mathrm{~m}$ and are resolved with 67 zones.

This calculation provides one test for the correctness of the solution of the data set, at least for the ADM mass of the spacetime for perturbative cases. This is valuable, because the code cannot be tested for the case when $K_{a b}=0$ since then the solution is trivially the MWBL solution.

As a general 3D strong field example we present in Table I data comparable to the A2B8 data set of [9]. Note that the data sets produced by the two methods are not 
TABLE I. Results for the A2B8 data set of [9]. All units are in terms of $m_{(2)}, h$ is the grid spacing, $n$ the number of grid points, $d$ is the residual, $\tau$ the truncation error, $\sigma$ the convergence rate, and $M_{\mathrm{ADM}}$ is the ADM mass of the box.

\begin{tabular}{cccccc}
\hline \hline \multicolumn{5}{c}{ A2B8, box of size 32} \\
\hline \multicolumn{5}{c}{$m_{(1)}=2$} & $m_{(2)}=1$ \\
& $\vec{r}_{(1)}=(0,0,4)$ & $\vec{r}_{(2)}=(0,0,-4)$ & \\
& $\vec{P}_{(1)}=(15,0,0)$ & $\vec{P}_{(2)}=(-15,0,0)$ & \\
& $\vec{S}_{(1)}=(-20,20,0)$ & $\vec{S}_{(2)}=(0,20,20)$ & \\
$h$ & $n$ & $\|d\|_{2}$ & $\|\tau\|_{2}$ & $\sigma$ & $M_{\mathrm{ADM}}$ \\
4.000 & $11^{3}$ & $1.159 e-08$ & $3.813 e-03$ & 4.029 & 9.209 \\
2.000 & $19^{3}$ & $5.350 e-08$ & $1.984 e-03$ & -2.119 & 10.097 \\
1.000 & $35^{3}$ & $2.141 e-07$ & $5.400 e-03$ & 0.838 & 10.601 \\
0.500 & $67^{3}$ & $2.582 e-07$ & $1.216 e-02$ & 1.121 & 10.833 \\
0.250 & $131^{3}$ & $1.552 e-07$ & $2.222 e-02$ & 1.430 & 10.917 \\
0.125 & $259^{3}$ & $6.629 e-08$ & $3.383 e-02$ & 1.712 & 10.942 \\
\hline \hline
\end{tabular}

identically the same. They are equally generic, but each has a different gravitational wave content.

There are two punctures with different mass parameters of order one, and with comparatively large values for boosts and spins. The results are obtained for a single box centered around the origin for various grid spacings. Each run was performed for the same multigrid parameters such that the values for the residua reflect the efficiency with which the discretized equations are solved at different resolutions. We chose to make the residua much smaller than the truncation error estimate, which turns out to be in a range compatible with the still rather large grid spacing. The convergence rates $\sigma$ are based on $u$ at three resolutions. As expected, we found in this and other examples that the convergence rate at the puncture points was one order less than elsewhere, but we also found that convergence was only affected very close to the punctures. As the resolution increases, the mass estimate converges with a rate comparable to $\sigma$.

To summarize, we have introduced a new setup for the initial data problem for multiple black holes with arbitrary mass, momentum, and spin in three dimensions. Comparing with the wormhole constructions of black holes, the key difference is how the various asymptotically flat regions are defined on a single copy of $R^{3}$. Instead of using an isometry condition at interior spheres, we remove the inner boundary by compactification and look for a solution to a modified Hamiltonian constraint equation on $R^{3}$, for which we have existence and uniqueness. A corresponding proof should be possible for the isometric wormhole data, although to our knowledge, such a proof has not been given yet.

There are two obvious numerical advantages compared to the wormhole approach. First, note that the solution $K^{a b}$ to the momentum constraint is all we need; i.e., since we do not impose the isometry condition there is no need for the method of images. Second, in the solution of the Hamiltonian constraint we avoid the numerical complications due to an inner boundary. Compared to the standard Cartesian (Cadez) method, one (two) levels of sophistication less are required for an implementation, which should make general black hole initial data more widely available.

Finally, note that black hole puncture data has already been successfully evolved in the case of 3D Schwarzschild initial data $[17,16]$, which is possible even when the punctures are part of the numerical grid since there are no physical singularities on the initial slice. For a long term evolution of black holes, one may start with data obtained by either the throat or puncture construction, and then cut out the interior regions at the apparent horizon to avoid the physical singularities on future slices $[12,18]$.

It is a pleasure to thank Helmut Friedrich, Alan Rendall, Bernd Schmidt, Ed Seidel, and Paul Walker for helpful discussions. The computations were performed at the AEI in Potsdam.

*Electronic address: brandt@aei-potsdam.mpg.de

${ }^{\dagger}$ Electronic address: bruegman@aei-potsdam.mpg.de

[1] A. Lichnerowicz, J. Math. 23, 3 (1944).

[2] J. York, in Sources of Gravitational Radiation, edited by L. Smarr (Cambridge University Press, Cambridge, 1979).

[3] A. Einstein and N. Rosen, Phys. Rev. 48, 73 (1935).

[4] J. Wheeler, Phys. Rev. 97, 511 (1955).

[5] C. Misner and J. Wheeler, Ann. Phys. (N.Y.) 2, 525 (1957).

[6] C. Misner, Ann. Phys. (N.Y.) 24, 102 (1963).

[7] D. Brill and R. Lindquist, Phys. Rev. 131, 471 (1963).

[8] J. York, in Frontiers in Numerical Relativity, edited by C. Evans, L. Finn, and D. Hobill (Cambridge University Press, Cambridge, 1989).

[9] G. Cook, M. Choptuik, M. Dubal, S. Klasky, R. Matzner, and S. Oliveira, Phys. Rev. D 47, 1471 (1993).

[10] H. Friedrich, Commun. Math. Phys. 119, 51 (1988).

[11] R. Beig and N. O'Murchadha, Classical Quantum Gravity 11, 419 (1994); 13, 739 (1996).

[12] J. Thornburg, Classical Quantum Gravity 4, 1119 (1987).

[13] M. Cantor, J. Math. Phys. (N.Y.) 20, 1741 (1979).

[14] D. Christodoulou and N. O'Murchadha, Commun. Math. Phys. 80, 271 (1981).

[15] A. Brandt, in Multigrid Methods, edited by W. Hackbusch and U. Trottenberg, Springer Lecture Notes in Mathematics No. 960 (Springer-Verlag, Berlin, 1982); W. Press, S. Teukolsky, W. Vetterling, and B. Flannery, Numerical Recipes in $C$ (Cambridge University Press, Cambridge, 1992).

[16] B. Brügmann, Phys. Rev. D 54, 7361 (1996).

[17] P. Anninos, K. Camarda, J. Massó, E. Seidel, W. Suen, and J. Towns, Phys. Rev. D 52, 2059 (1995).

[18] E. Seidel and W. Suen, Phys. Rev. Lett. 69, 1845 (1992). 